\title{
Evidence supporting the benefits of marijuana for Crohn's disease and ulcerative colitis is extremely limited: a meta-analysis of the literature
}

\author{
Anna Desmarais, Stephen Smiddy, Sneha Reddy, Mohammed El-Dallal, Jonathan Erlich, \\ Joseph D. Feuerstein
}

Beth Israel Deaconess Medical Center, Boston, MA, USA

\section{Abstract}

\begin{abstract}
Background Medical marijuana is increasingly used to control inflammation and pain in inflammatory bowel disease (IBD). We performed a meta-analysis to investigate the effect of marijuana on the clinical response, induction of clinical remission, and maintenance of clinical remission compared to placebo/standard of care.
\end{abstract}

Methods We performed a systematic search of PubMed, Embase, and Web of Science in June 2019, for cannabis/marijuana and IBD, Crohn's disease or ulcerative colitis (UC). The statistical analysis was performed using Revman (version 5.3). GRADE methodology was used to assess the quality of the evidence.

Results Of the 334 studies initially reviewed, 1 trial in UC and 2 trials in Crohn's disease met eligibility. For UC, 29 patients were treated with marijuana and 31 with placebo/standard of care. There was no difference in failure to achieve clinical remission (relative risk [RR] 1.02, 95\% confidence interval [CI] 0.76-1.37) or response (RR 0.99, 95\%CI 0.65-1.21). Adverse events occurred in all patients receiving marijuana (RR 1.28, 95\%CI 1.05-1.56). For Crohn's disease, 21 patients were treated with marijuana and 19 with placebo/standard of care. There was no difference in failure to achieve clinical remission (RR $0.72,95 \% \mathrm{CI} 0.47-1.12$ ) or failure to achieve clinical response (RR $0.15,95 \% \mathrm{CI} 0.02-1.05$ ). Adverse events were not reported per patient. The quality of evidence was low to very low using GRADE methodology.

Conclusion Data supporting the use of marijuana for the management of IBD are extremely limited. Further well-designed studies are needed before any positive conclusions regarding marijuana use can be drawn.

Keywords Marijuana, cannabis, ulcerative colitis, Crohn's disease, inflammatory bowel disease

Ann Gastroenterol 2020; 33 (4): 1-5

\section{Introduction}

The potential benefit of marijuana in the treatment of different diseases and pain has been of great intrigue. In recent years, over 37 states have moved to legalize the use of medical

Division of Gastroenterology, Beth Israel Deaconess Medical Center, Boston, MA, USA

Conflict of Interest: None

Correspondence to: Joseph D. Feuerstein, 110 Francis St 8e

Gastroenterology Division of Gastroenterology, Beth Israel Deaconess Medical Center, 110 Francis St 8e Gastroenterology, Boston MA02215

USA, e-mail: jfeuerst@bidmc.harvard.edu

Received 1 April 2020; accepted 12 May 2020;

published online 22 June 2020

DOI: https://doi.org/10.20524/aog.2020.0516 marijuana to treat specific conditions and ailments [1]. The determination of conditions for which medical marijuana is approved is not based on a review of evidence by the Food and Drug Administration (FDA), but is made by state regulatory organizations that regulate the use of medical marijuana [2].

The evidence supporting marijuana has been of variable provenance $[2,3]$. Recently a marijuana derivative was approved for the treatment of a rare type of seizure disorder [4,5]. To gain this approval, appropriate phase 1-3 studies were performed and their success allowed submission of the drug to the FDA for authorization. In contrast, while Crohn's disease is considered an acceptable indication for the use of medical marijuana, the evidence supporting its benefits is more limited and no phase 1-3 studies have been performed [6,7].

In survey data of patients in inflammatory bowel disease clinics, the use of marijuana for treatment of both Crohn's disease and ulcerative colitis is common $[8,9]$. Many patients 
self-report an improvement in symptoms and control of pain [7-9]. In patients with more severe disease the selfreported benefits were more prominent. However, objective data supporting these findings are limited.

Studies by Naftali et al have shown that patients prescribed marijuana derivatives may show some improvement, but the benefit stops short of achieving a response or inducing disease remission [10-12]. In these studies, a majority of patients were also taking standard therapy; thus, evidence to support any use of marijuana or its derivatives as a replacement for standard therapy is even more limited [10-12]. Therefore, we performed a metaanalysis of the current evidence for the use of marijuana to induce disease remission or achieve a response in patients with ulcerative colitis or Crohn's disease.

\section{Materials and methods}

\section{Data source and literature search}

A comprehensive literature search was performed with the assistance of a medical librarian at Harvard Medical School who has expertise in meta-analysis searches. The literature was searched for randomized and non-randomized trials investigating the efficacy of marijuana use compared to placebo/standard therapy in terms of response and induction of remission in patients with ulcerative colitis or Crohn's disease. Databases searched included PubMed, EMBASE and Web of Science. A Google search was also performed to assess any missing studies not indexed in the above datasets. The search strategy is detailed in Supplementary Table 1. The initial search was performed on June 15, 2019.

\section{Study selection}

\section{Inclusion criteria}

Studies were included if they involved the use of marijuana compared to placebo/standard of care in patients with ulcerative colitis or Crohn's disease and evaluated the response to therapy and/or induction of remission. Both randomized and non-randomized studies were included.

\section{Exclusion criteria}

Non-human studies, survey studies and studies that did not have a comparator arm were excluded.

\section{Data extraction}

Three reviewers (AD, SM and SR) independently reviewed all search results. Initial results were reviewed by title to assess if a study was potentially eligible for inclusion. If a screened title was considered eligible or potentially eligible then the abstract was reviewed in detail. Any disagreement was resolved by the evaluation of a fourth reviewer (JDF).

\section{Outcome measures}

The primary outcome of this study was to assess the response to therapy and induction of remission when using marijuana or its derivatives compared to placebo/standard of care therapy. A secondary outcome was the rates of adverse events related in patients using marijuana or its derivatives compared to placebo/standard of care therapy.

\section{Risk of bias assessment and quality of evidence assessment}

Risk of bias and quality of evidence were assessed using the Grading of Recommendations Assessment, Development, and Evaluation (GRADE) approach [13]. The overall certainty in the evidence was assessed using the GRADE domains, including risk of bias, inconsistency, indirectness, impression, and other publication bias considerations. Evidence was then graded as high, moderate, low or very low.

\section{Statistical analysis}

Comparative studies were analyzed using pooled relative risk (RR) and 95\% confidence intervals (CI) were calculated based on the DerSimonian-Laird random-effects model. Heterogeneity was assessed using the $I^{2}$ statistic, with values $>50 \%$ suggesting significant heterogeneity. Statistical analysis was performed using RevMan, version 5.3 (Cochrane Collaboration, Copenhagen, Denmark) and GradePro/GDT software (McMaster University, 2015) $[14,15]$. The review was performed using the standards set forth by the Preferred Reporting Items for Systematic Reviews and Meta-analysis (PRISM) statement.

\section{Results}

The search of PubMed, Embase and Web of Science resulted in 334 articles (Fig. 1). After removal of duplicates, 263 potential studies were reviewed in depth, of which 26 were reviewed in further detail, resulting in 3 studies remaining in the final qualitative and quantitative analysis. Only one study met the inclusion criteria for ulcerative colitis and 2 for Crohn's disease [10,16,17]. In total, 29 patients with ulcerative colitis were treated with marijuana, of whom 6 were female; these patients had a mean Mayo score of 6.9 at the time of enrollment. Irving et al used a hard gelatin capsule containing $50 \mathrm{mg}$ of cannabidiol (CBD)-rich botanical extract, started at one pill b.i.d. up to a peak dosage of 5 pills b.i.d. Patients were followed for 12 weeks [16]. In Crohn's disease 21 patients were treated with marijuana. Naftali et al 


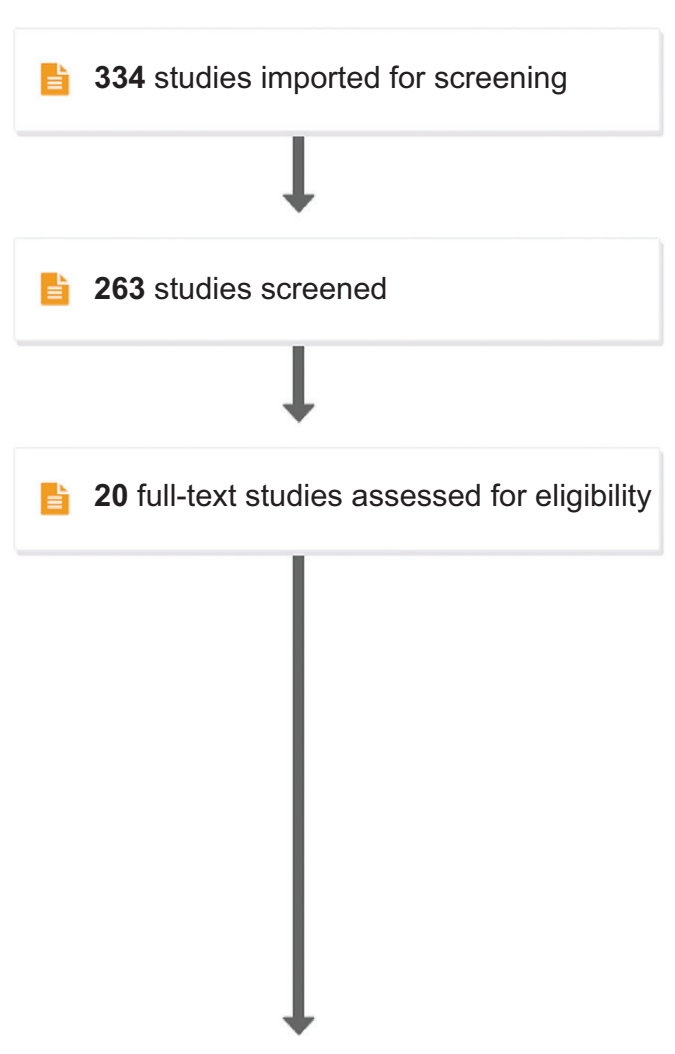

3 studies included

Figure 1 PRISMA flow diagram

(2013) included 11 patients with a Crohn's disease activity index $(\mathrm{CDAI})>200$ in their marijuana arm, of whom 5 were female. They used cigarettes with tetrahydrocannabinol (THC) extracted from a cannabis flower [10]. Naftali et al (2017) included 10 patients with a CDAI score of $>200$ in their marijuana arm, of whom 4 were female [17]. They used a CBD extract from Lebanese hashish, which contains 5\% CBD. It was weight-based dosing, with $0.3 \mathrm{mg} / \mathrm{kg}$ of CBD extract mixed $5 \mathrm{mg} / \mathrm{mL}$ with olive oil [17]. Both studies followed their patients for 8 weeks.

\section{Ulcerative colitis outcomes}

Only the study by Irving et al met the criteria for inclusion [16]. Of the 29 patients treated with marijuana, 22 failed to achieve remission, compared to 23 of the 31 patients receiving placebo (RR 1.02, 95\%CI 0.76-1.37). Similarly, 20/29 patients receiving marijuana and $24 / 31$ patients receiving placebo failed to achieve a response to therapy (RR 0.89, 95\%CI 0.65-1.21). No studies assessed the use of marijuana for maintenance of remission. Adverse events were common in the marijuana group, with all 29 patients reporting some adverse event, compared to $24 / 31$ patients in the placebo group (RR $1.28,95 \%$ CI 1.05-1.56).
71 duplicates removed

236 studies irrelevant

\section{7 studies excluded}

7 Wrong study design

4 Survey study

2 Abstract of full study that is

already included

2 Wrong outcomes

1 Duplicate abstract

1 Not applicable study

\section{Crohn's disease outcomes}

Two studies by Naftali et al $(2013,2017)$ were included in the assessment of induction of remission [10,17]. Twelve of 21 patients did not achieve induction of remission when using marijuana or its derivative, compared to $15 / 19$ in the placebo arm (RR 0.72, 95\%CI 0.47-1.12) (Fig. 2). Regarding the response to therapy, only Naftali 2013 was included, with only one patient failing to respond $(n=1 / 11)$ compared to $6 / 10$ in the placebo arm (RR $0.15,95 \%$ CI $0.02-1.05$ ). No studies assessed the use of marijuana for maintenance of remission. Adverse events were not reported on a per patient basis and therefore could not be determined.

\section{GRADE quality of evidence assessment}

The quality of the evidence was considered low for all the outcomes in ulcerative colitis, owing to imprecision from the very low patient sample size and the confidence intervals intersecting the line of unity. For Crohn's disease it was further downgraded to very low, because of the heterogeneity of the different formulations of the marijuana used in the study designs. 


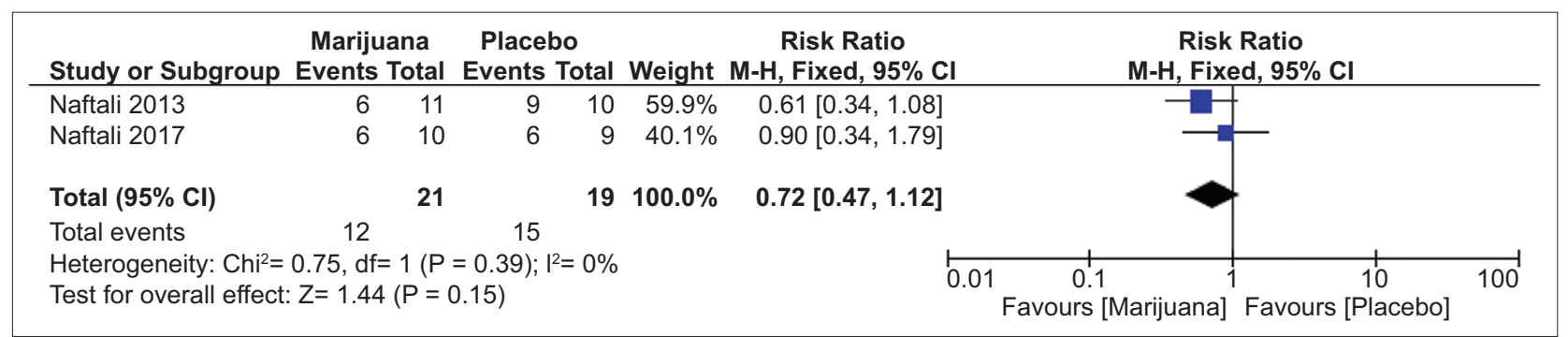

Figure 2 Failure to achieve remission in Crohn's disease

\section{Discussion}

The challenge of trying to find another drug that is safe and efficacious to manage patients with inflammatory bowel disease remains a key focus and one that patients eagerly yearn for. Public perception is that marijuana is safe, with limited side-effects. Given its potential to treat pain and the fact that patients with inflammatory bowel disease can have pain, it is not surprising to see interest in utilizing marijuana and its derivatives to treat Crohn's and ulcerative colitis. However, this meta-analysis highlights the very limited evidence available to support the use of marijuana in inflammatory bowel disease. In ulcerative colitis, only one study could be included; this did not find any benefit of marijuana but did report side-effects from its use in all patients. Similarly, in Crohn's disease only 2 studies were included, but no benefits were seen in terms of disease response or remission.

Given that marijuana is not an FDA-approved drug, but is approved for use as a medical treatment by state regulators, the burden of therapeutic efficacy is not what is traditionally required by the FDA $[1,3]$. Groups with expertise in marijuana recommended the conditions for which it should be approved, and these were readily adopted [1]. However, this study highlights that there is probably no evidence-based role for medical marijuana in the treatment of ulcerative colitis or Crohn's disease at this time. Based on our evaluation of the evidence, neither disease showed any improvement in response or remission. The use of marijuana as a drug to treat inflammatory bowel disease still requires much larger, welldesigned drug trials [18]. In the current state, evidence-based medicine would recommend against its use as a treatment, given the lack of efficacy shown in these studies.

While marijuana may not be effective as a pharmacologic therapy for disease management, it may have a role as an adjunct to standard therapy [18]. Some studies have reported improvements in pain and overall improved disease outlook when patients took marijuana or its derivatives $[6,7,12]$. In surveys of patients with inflammatory bowel disease, those who used marijuana had a better overall quality of life compared to those who did not $[6,8]$. However, while this subjective reporting of improvement in pain and symptoms is of interest, the most efficacious way to treat pain and other symptoms in inflammatory bowel disease is to treat the underlying inflammation [19]. Naftali et al did report improvements in pain, despite a lack of improvement in response or remission. This raises concerns similar to those regarding opiate use in inflammatory bowel disease: that the drug might mask symptoms that indicate the failure of drug therapy, inadvertently resulting in worse disease outcomes [20]. Without more data regarding the safety of marijuana, caution is needed when using it to avoid disease-related complications.

The preclinical data suggested that cannabis-based medications could have anti-inflammatory effects via multiple pathways, including T-cell apoptosis, downregulate cytokine and chemokine production, upregulate T-regulatory cells, among others $[1,2]$. However, the clinical data lead to less optimistic findings. There have been multiple studies that examined the effects of cannabis on patients with multiple sclerosis, with conflicting results. While some randomized trials showed that cannabis products provide relief from spasticity $[3,4]$, others showed less promising effects $[5,6]$.

Similarly, despite the anti-inflammatory effects seen in animal and in vitro models of cannabis-based medications used to treat rheumatoid arthritis and systemic sclerosis, there is no published clinical trial to date to prove these theoretical benefits [7]. Nonetheless, Blake et al showed that cannabis could help with pain management in patients with rheumatoid arthritis when compared to placebo [8].

Despite the public perception that marijuana is safe, with few side-effects, even in these small trials there were high rates of adverse events in the marijuana therapy arms [6,7]. While serious complications were not seen, the duration of therapy was limited [7]. There were no studies reporting on the use of maintenance of remission, which would require a longer treatment duration and could potentially result in more severe adverse events. Additionally, the studies included in this analysis used marijuana with different doses, routes, potency and frequency $[10,16]$. This all may factor into the risk profile of the medication in both the short and long term. It also complicates the ability to make a recommendation to patients who want to use marijuana, regarding the formulation, dose and frequency with which it should be taken. Ultimately, more data are needed to better evaluate the risk profile of a standardized formulation of marijuana in the short and long term.

Our study has a number of limitations. Only a few studies were available that investigated the response and induction of remission in ulcerative colitis and Crohn's disease, and the total number of patients evaluated was small. However, this is also a strength of the study, in that it highlights the limited data supporting the use of marijuana in the treatment of inflammatory bowel disease. Additionally, there was no standardization of the dose, route or frequency of marijuana use in the studies, making 
it challenging to make a recommendation to patients on how to take the drug. This is an important limitation of the study, but also a strength that highlights the heterogeneity of the data regarding the use of marijuana.

The current literature does not support the use of marijuana or its derivatives in the treatment of ulcerative colitis or Crohn's disease for induction of remission or response to therapy. Ultimately more studies are needed to standardize the dose, frequency, potency and route of administration of marijuana in relation to the response to therapy, induction of remission and maintenance of remission. Until such studies are performed, one should consider the use of marijuana for inflammatory bowel disease as experimental and with unclear benefits.

\section{Summary Box}

\section{What is already known:}

- There is a potential benefit of using marijuana in treating inflammatory bowel disease

- Over 37 states have moved to legalize the use of medical marijuana to treat specific conditions, based on regulatory measures rather than a review of evidence

- The use of medical marijuana among patients with inflammatory bowel disease is common

\section{What the new findings are:}

- There are limited number of small studies that examined the effect of using marijuana in treating inflammatory bowel disease

- These studies used different doses, routes, potency and frequency of marijuana

- At present, there is no evidence to support the use of medical marijuana in treating inflammatory bowel disease

\section{References}

1. Map of Marijuana Legality by State. Available from: https://disa. com/map-of-marijuana-legality-by-state [Accessed 24 May 2020].
2. Wilkinson ST, D'Souza DC. Problems with the medicalization of marijuana. JAMA 2014;311:2377-2378.

3. D'Souza DC, Ranganathan M. Medical marijuana: is the cart before the horse? JAMA 2015;313:2431-2432.

4. Rubin R. The Path to the First FDA-approved cannabis-derived treatment and what comes next. JAMA 2018;320:1227-1229.

5. O'Connell BK, Gloss D, Devinsky O. Cannabinoids in treatmentresistant epilepsy: a review. Epilepsy Behav 2017;70(Pt B):341-348.

6. Swaminath A, Berlin EP, Cheifetz A, et al. The role of cannabis in the management of inflammatory bowel disease. Inflamm Bowel Dis 2018;25:427-435.

7. Cheifetz AS, Gianotti R, Luber R, Gibson PR. Complementary and alternative medicines used by patients with inflammatory bowel diseases. Gastroenterology 2017;152:415-429.

8. Merker AM, Riaz M, Friedman S, Allegretti JR, Korzenik J. Legalization of medicinal marijuana has minimal impact on use patterns in patients with inflammatory bowel disease. Inflamm Bowel Dis 2018;24:2309-2314.

9. Pi S, Rosenfeld G, Enns R, et al. Patterns and motivations of cannabis use amongst patients with inflammatory bowel disease. GastroHep 2019;1:100-107.

10. Naftali T, Bar-Lev Schleider L, Dotan I, Lansky EP, Sklerovsky Benjaminov F, Konikoff FM. Cannabis induces a clinical response in patients with Crohn's disease: a prospective placebo-controlled study. Clin Gastroenterol Hepatol 2013;11:1276-1280.

11. Naftali T, Lev LB, Yablecovitch D, Half E, Konikoff FM. Treatment of Crohn's disease with cannabis: an observational study. Isr Med Assoc J 2011;13:455-458.

12. Naftali T, Mechulam R, Lev LB, Konikoff FM. Cannabis for inflammatory bowel disease. Dig Dis 2014;32:468-474.

13. Harbour R, Miller J. A new system for grading recommendations in evidence based guidelines. BMJ 2001;323:334-336.

14. GRADEpro G. GRADEpro guideline development tool [software]. McMaster University 2015;435.

15. Collaboration C. Review Manager (RevMan).[5.3.5]. Oxford; 2015.

16. Irving PM, Iqbal T, Nwokolo C, et al. A randomized, double-blind, placebo-controlled, parallel-group, pilot study of cannabidiol-rich botanical extract in the symptomatic treatment of ulcerative colitis. Inflamm Bowel Dis 2018;24:714-724.

17. Naftali T, Mechulam R, Marii A, et al. Low-dose cannabidiol is safe but not effective in the treatment for Crohn's disease, a randomized controlled trial. Dig Dis Sci 2017;62:1615-1620.

18. Naftali T. Is cannabis of potential value as a therapeutic for inflammatory bowel disease? Springer; 2019.

19. Feuerstein JD, Cheifetz AS. Crohn disease: epidemiology, diagnosis, and management. Mayo Clin Proc 2017;92:1088-1103.

20. Colombel JF, Shin A, Gibson PR. AGA clinical practice update on functional gastrointestinal symptoms in patients with inflammatory bowel disease: Expert review. Clin Gastroenterol Hepatol 2019;17:380-390. 


\section{Supplementary material}

Supplementary Table 1 Search criteria

Search criteria

Number of results

\begin{tabular}{|c|c|c|}
\hline PubMed & $\begin{array}{l}\text { (((((((“ulcerative colitis") OR UC) OR “inflammatory bowel disease”) OR IBD) OR “crohn”) OR "crohns") } \\
\text { OR “crohn's”)) AND ((((“cannabis”) OR CBD) OR THC) OR “marijuana”) }\end{array}$ & 200 \\
\hline Embase & $\begin{array}{l}\text { ('cannabinoid'/exp OR 'cannabinoid' OR 'cannabis'/exp OR 'cannabis') AND ('inflammatory bowel disease'/ } \\
\text { exp OR 'inflammatory bowel disease' OR 'ulcerative colitis'/exp OR 'ulcerative colitis' OR 'crohn disease'/exp } \\
\text { OR 'crohn disease') }\end{array}$ & 408 \\
\hline $\begin{array}{l}\text { Web of } \\
\text { Science }\end{array}$ & $\begin{array}{l}\# 3 \quad 114 \\
\# 2 \text { AND \# } 1 \\
\text { Indexes=SCI-EXPANDED, SSCI, A\&HCI, CPCI-S, CPCI-SSH, BKCI-S, BKCI-SSH, ESCI, CCR- } \\
\text { EXPANDED, IC Timespan=All years } \\
\text { \# } 2 \quad 148,195 \\
\text { ALL = (Inflammatory Bowel Disease OR Ulcerative colitis OR Crohn }{ }^{\star} \text { ) } \\
\text { Indexes=SCI-EXPANDED, SSCI, A\&HCI, CPCI-S, CPCI-SSH, BKCI-S, BKCI-SSH, ESCI, CCR- } \\
\text { EXPANDED, IC Timespan=All years } \\
\text { \# } 1 \quad 33,585 \\
\text { ALL = (Marijuana OR Cannabis) } \\
\text { Indexes=SCI-EXPANDED, SSCI, A\&HCI, CPCI-S, CPCI-SSH, BKCI-S, BKCI-SSH, ESCI, CCR- } \\
\text { EXPANDED, IC Timespan=All years }\end{array}$ & 114 \\
\hline
\end{tabular}

